IIIIIIIIIIIIIIIIIIIIIIIIIIIIIIIIIIII

Original Article

IIIIIIIIIIIIIIIIIIIIIIIIIIIIIIIIII

\title{
Determination of nitenpyram and its metabolites in agricultural products by using hydrophilic interaction liquid chromatography-tandem mass spectrometry
}

\author{
Tatsuo Yoshida, ${ }^{1,2, *}$ Hiroshi Murakawa, ${ }^{1}$ and Kei Toda ${ }^{2}$ \\ ${ }^{1}$ Kumamoto Prefectural Institute of Public Health and Environmental Science, \\ 1240-1 Kurisaki, Uto, Kumamoto 869-0425, Japan \\ ${ }^{2}$ Department of Chemistry, Kumamoto University, 2-39-1 Kurokami, Kumamoto 860-8555, Japan
}

(Received August 26, 2012; Accepted December 14, 2012)

\begin{abstract}
A simple and rapid method for the measurement of a neonicotinoid pesticide, nitenpyram, and its metabolites, CPMA and CPMF, was investigated. The three compounds were determined individually and simultaneously by simple ultrasonic extraction followed by hydrophilic interaction liquid chromatography-tandem mass spectrometry (HILIC-MS/MS). Good chromatograms were obtained using a non-modified silica gel column. The calibration curves were straight $\left(R^{2}>0.999\right)$ for each compound; they were $0.002-2 \mathrm{mg} / \mathrm{kg}$ for $5 \mathrm{~g}$ of agricultural product sample with limits of detection $(S / N=3)$ of $0.24-0.36 \mu \mathrm{g} / \mathrm{kg}$ (nitenpyram), $0.12-0.19 \mu \mathrm{g} / \mathrm{kg}$ (CPMA), and $0.13-0.18 \mu \mathrm{g} / \mathrm{kg}$ (CPMF). Good recoveries were obtained for sweet pepper, mandarin orange, onion, and brown rice (93.0-106.6\% in recovery). For contaminated samples, data by the presented method agreed well with nitenpyram data by HPLC-UV and the total of CPMA and CPMF by derivatization followed by gas chromatography-flame thermionic detection.

Keywords: nitenpyram, 2-[N-(6-chloro-3-pyridylmethyl)- $N$-ethyl]amino-2-methyliminoacetic acid (CPMA), $N$ (6-chloro-3-pyridylmethyl)- $N$-ethyl- $N^{\prime}$-methylformamidine (CPMF), hydrophilic interaction liquid chromatography, tandem mass spectrometry.
\end{abstract}

\section{Introduction}

Some pesticides have high activity even after metabolizing; thus, both the pesticides and their metabolites must be analyzed. The Japanese Ministry of Health, Labor, and Welfare determined as a food standard that the nitenpyram content in food products must be given together with that of its metabolites, namely, 2-[N-(6-chloro-3-pyridylmethyl)$\mathrm{N}$-ethyl]amino-2-methyliminoacetic acid (CPMA) and $\mathrm{N}$ (6-chloro-3-pyridilmethyl)- $N$-ethyl- $N^{\prime}$-methylformamidine (CPMF). ${ }^{1)}$ In the official analytical method of the Japanese Ministry of Health, Labor, and Welfare, CPMA and CPMF are first converted to $\mathrm{N}$-(6-chloro-3-pyridilmethyl)- $\mathrm{N}$-ethylformamide (CPF) and determined by GC, and those contents are added to the concentration of nitenpyram and not given separately. Furthermore, the method is complicated due to a troublesome procedure that requires use of many reagents and a purification column.

Another problem to determine CPMA and CPMF discrimi-

\footnotetext{
* To whom correspondence should be addressed.

E-mail: yoshida-t-dp@pref.kumamoto.lg.jp

Published online February 14, 2013

(c) Pesticide Science Society of Japan
}

natively is that CPMA tends to change to CPMF in an organic solvent, such as acetone and ethylacetate. ${ }^{2)}$ Organic solvent extraction and subsequent concentration are usually adopted in the analytical procedure for organic compounds. Tsumura et al. reported that $80.4 \%$ of CPMA in a water/acetone $(20: 150)$ mixture was converted to CPMF during rotary evaporator concentration and recovery was only 9.0\%. ${ }^{3)}$ In addition, CPMA is highly polar, ${ }^{2,3)}$ and a chromatogram peak is not as good in shape as twin peaks in separation by common reversed-phase chromatography. ${ }^{4)}$ Consequently, a novel analytical procedure is required for the measurement of CPMA that would be entail extraction with a lower-ratio-organic solvent and would not require a concentration procedure. A separation process is also needed to investigate to handle the relatively highly polar compounds.

We investigated extractions of pesticides from many kinds of agricultural products using an acetonitrile/water mixture ${ }^{5)}$ in which a relatively hydrophilic medium was used and preconcentration for analysis was not needed. Recently, hydrophilic interaction liquid chromatography (HILIC) has become popular to analyze highly polar compounds. ${ }^{6-11)}$ HILIC is a kind of normalphase chromatography which entails use of a water/organic solvent mixture eluent and a stationary phase having higher polarity than the mobile phase. Whereas a non-polar organic solvent 
is used for conventional normal-phase chromatography and handling of hydrophilic compounds is normally difficult, CPMA is expected to have good affinity for both the mobile and stationary phases of the HILIC system.

In this work, a novel method was investigated to measure nitenpyram, CPMA, and CPMF simply and discriminatively using HILIC. As CPF is not produced under natural conditions, ${ }^{3)}$ the targets for analysis were the other three compounds. A watermixed solvent was selected for the extraction to prevent conversion of CPMA to CPMF. Using the optimal extraction medium, a recovery test was performed by standard addition to agricultural samples. The presented HILIC-MS/MS was applied to the measurement of the three compounds, and the obtained data were compared to those by the official analytical method of the Japanese Ministry of Health, Labor, and Welfare, ${ }^{1)}$ which requires two methods: HPLC-UV for nitenpyram and gas chromatography-flame thermionic detection (GC-FTD) for the total of CPMA and CPMF.

\section{Materials and Methods}

\section{Standard solution}

Nitenpyram, CPMA, CPMF, and CPF were obtained from Wako Pure Chemical Industries (Osaka, Japan). CPMA was dissolved in water, and the other compounds were dissolved in methanol to obtain $500 \mathrm{mg} / \mathrm{L}$ stock solutions.

\section{Sample treatment}

The effect of water content in the extraction solvent was examined using asparagus containing nitenpyram, CPMA, and CPMF. Recovery was examined with sweet pepper, mandarin orange (pulp without peel), onion, and brown rice; all were confirmed not to contain nitenpyram or any metabolites. Brown rice was pulverized using a food cutter into particles that could be passed through a $425 \mu \mathrm{m}$ standard sieve, and the other vegetables/fruit were prepared using a food cutter and a homogenizer, then, each of the three nitenpyram compounds was added to be 0.005 or $0.5 \mathrm{mg} / \mathrm{kg}$ for the recovery tests. Two asparagus samples and a melon (pulp without peel) sample containing nitenpyram and the metabolites were used for the evaluation of contaminated samples analysis.

The extraction process is shown in Fig. 2. Test samples $(5 \mathrm{~g}$ each) prepared as mentioned above were treated in $20 \mathrm{~mL}$ of an acetonitrile-water mixture, typically, 65:35, with the help of ultrasonication (Nissei Ultrasonic Cleaner NS-605, 600W, $28 \mathrm{kHz}$, Nihonseiki Kaisha Ltd., Tokyo, Japan) for $5 \mathrm{~min}$; then, they were centrifuged for $5 \mathrm{~min}$ at $1882 \times \mathrm{g}$. The solid was treated with the same solvent one more time in the same way, and the top clear layers of the first and second extractions were collected and mixed with an acetonitrile-water mixture to obtain a volume of $50 \mathrm{~mL}$. The solution was filtered with a hydrophobic $0.45 \mu \mathrm{m}$ filter (Chromatodisk 25N, GL Sciences, Tokyo, Japan) for chromatographic analysis. The calibration curve was obtained using matrix-matched standard solutions prepared using an extracted solution from contaminant-free samples.

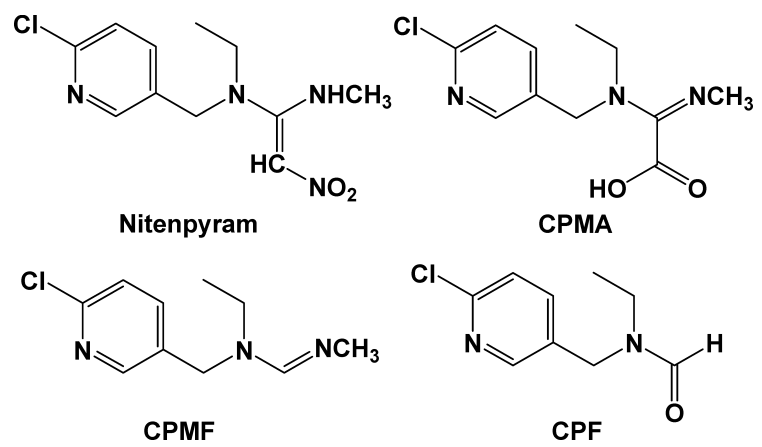

Fig. 1. Chemical structures of nitenpyram, CPMA, CPMF, and CPF.

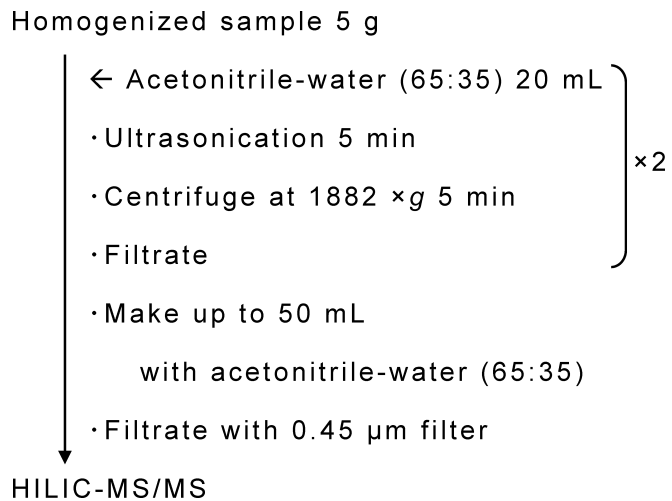

Fig. 2. Sample treatment for HILIC-MS/MS analysis.

\section{HILIC-MS/MS analysis for nitenpyram, CPMA, and CPMF} The instrument used in this analysis was an HPLC (Waters 2795, Waters, MA) coupled with a tandem mass spectrometer (Quattro Premier, Waters). The separation column was a non-modified silica gel column (Atlantis HILIC, $4.6 \mathrm{~mm}$ i.d. $\times 150 \mathrm{~mm} \mathrm{~L}$, $3 \mu \mathrm{m}$, Waters). In addition, the amide type (TSKgel Amide 80, $2.0 \mathrm{~mm}$ i.d. $\times 150 \mathrm{mmL}, 3 \mu \mathrm{m}$, Waters), the sulfobetaine type (ZIC-pHILIC, $4.6 \mathrm{~mm}$ i.d. $\times 150 \mathrm{~mm} \mathrm{~L}, 5 \mu \mathrm{m}$, Merck KGaA, Darmstadt, Germany), the diol type (Inersil HILIC, $4.6 \mathrm{~mm}$ i.d. $\times 150 \mathrm{~mm} \mathrm{~L}, 3 \mu \mathrm{m}$, GL Sciences), and the triazole type (COSMOSIL HILIC, $3.0 \mathrm{~mm}$ i.d. $\times 150 \mathrm{~mm} \mathrm{~L}, 5 \mu \mathrm{m}$, Nacalai Tesque, Kyoto, Japan) were tested for comparison of HILIC columns.

The sample injection was $10 \mu \mathrm{L}$ in volume, the column temperature was $40^{\circ} \mathrm{C}$, and the eluent was distilled water-acetonitrile-500 $\mathrm{mM}$ ammonium acetate $(25: 70: 5)$. The flow rate used for the amide type $(2.0 \mathrm{~mm}$ i.d.) was $0.1 \mathrm{~mL} / \mathrm{min}, 0.2 \mathrm{~mL} / \mathrm{min}$ for the triazole type $(3.0 \mathrm{~mm}$ i.d.), and $0.5 \mathrm{~mL} / \mathrm{min}$ for the other columns ( $4.6 \mathrm{~mm}$ i.d.). Each analyte was ionized by electrospray ionization (ESI) in the positive mode, and the quantitative measurement was performed by selected reaction monitoring (SRM). During the ESI process, the capillary voltage was $3.45 \mathrm{kV}$, the source temperature was $120^{\circ} \mathrm{C}$, the desolvation temperature was $350^{\circ} \mathrm{C}$, and the total nitrogen flow and cone flow rate were $1000 \mathrm{~L} / \mathrm{h}$ and $100 \mathrm{~L} / \mathrm{h}$, respectively. 
Table 1. Chromatographic and SRM parameters for nitenpyram, CPMA, and CPMF

\begin{tabular}{|c|c|c|c|c|c|c|c|c|c|}
\hline Compound & $t_{\mathrm{R}},{ }^{a)} \min$ & Ion type & $\begin{array}{l}\text { Quantification } \\
\text { transition }\end{array}$ & Cone, $\mathrm{V}$ & $\mathrm{CE},{ }^{b)} \mathrm{eV}$ & $\begin{array}{l}\text { Confirmatory } \\
\text { transition }\end{array}$ & Cone, $\mathrm{V}$ & $\mathrm{CE},{ }^{b)} \mathrm{eV}$ & Ion ratio \\
\hline Nitenpyram & 4.6 & {$[\mathrm{M}+\mathrm{H}]^{+}$} & $270.66 \rightarrow 125.62$ & 25 & 25 & $270.66 \rightarrow 188.78$ & 25 & 25 & 13.6 \\
\hline CPMA & 5.1 & {$[\mathrm{M}+\mathrm{H}]^{+}$} & $256.18 \rightarrow 126.12$ & 25 & 25 & $256.18 \rightarrow 176.45$ & 25 & 10 & 2.3 \\
\hline CPMF & 9.4 & {$[\mathrm{M}+\mathrm{H}]^{+}$} & $212.30 \rightarrow 126.33$ & 35 & 20 & $212.30 \rightarrow 90.47$ & 35 & 35 & 4.6 \\
\hline
\end{tabular}

${ }^{a)}$ Retention time, ${ }^{b}$ collision energy.
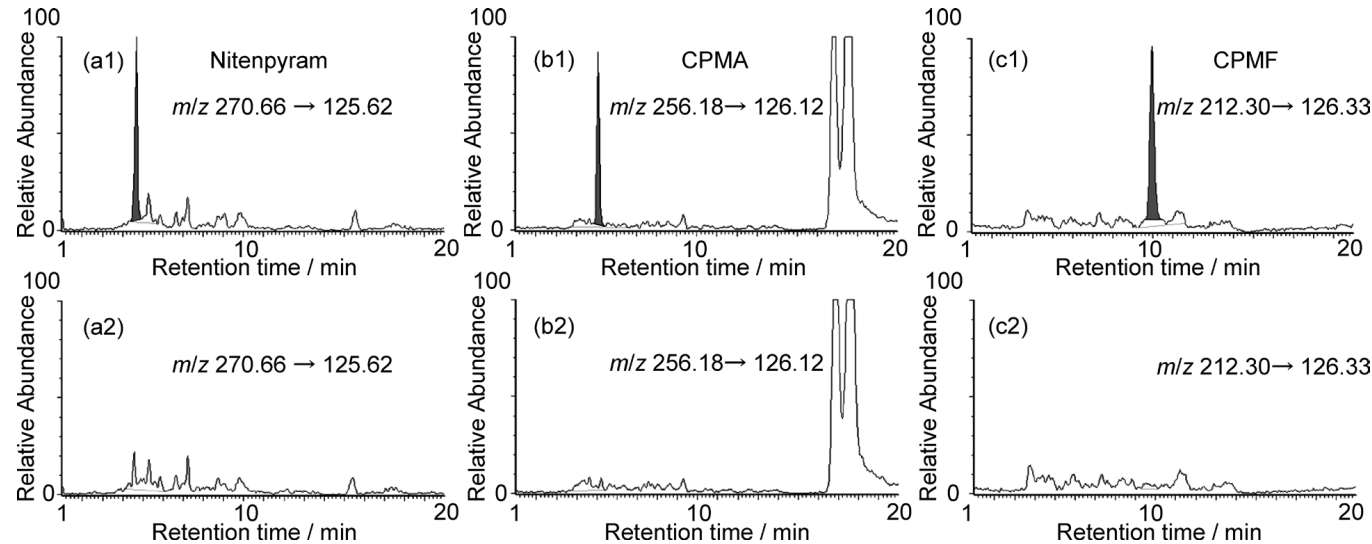

Fig. 3. Chromatograms obtained for extracts from sweet pepper spiked with $0.005 \mathrm{mg} / \mathrm{kg}$ nitenpyram (a1), CPMA (b1), and CPMF (c1) and sweet pepper extract (a2), (b2), and (c2)

\section{Nitenpyram analysis by HPLC-UV}

Nitenpyram was also analyzed using HPLC-UV according to the standard method. A 5-g agricultural product sample was homogenized with $20 \mathrm{~mL}$ of acetone and filtered through glass wool. The treatment was repeated once. Both filtrates were gathered and concentrated using a rotary evaporator. The solution was then placed on the head of a column packed with porous diatomaceous earth (InertSep K-solute $20 \mathrm{~mL}$, GL Sciences); $15 \mathrm{~min}$ later $50 \mathrm{~mL}$ of hexane was added to the column, and the eluent was wasted. Then, $50 \mathrm{~mL}$ of diethyl ether-hexane mixture $(1: 1)$ and $50 \mathrm{~mL}$ of dichloromethane were successively introduced into the column, and the eluent was transferred to another column $(15 \mathrm{~mm}$ i.d. $\times 300 \mathrm{~mm} \mathrm{~L})$ packed with $10 \mathrm{~g}$ of silica gel (for gas chromatography use, 63-200 $\mu \mathrm{m}$, Merck KGaA) which was sintered in dichloromethane before use. The sample-loaded column was washed with $50 \mathrm{~mL}$ of an acetone-dichloromethan mixture (1:1), and the compounds were then eluted with $50 \mathrm{~mL}$ of acetone. The eluent was concentrated using a rotary evaporator, and a methanol-50 $\mathrm{mM}$ potassium dihydrogen phosphate mixture $(3: 17)$ was added to achieve a volume of $2 \mathrm{~mL}$ for HPLC analysis.

The HPLC system used comprised a high-pressure pump (LC-10AD), a column oven (CTO-10A), and a UV detector (SPD-M10Avp), all purchased from Shimadzu, Kyoto, Japan. Ten microliters of sample was injected into a carrier stream consisting of a methanol-50 mM potassium dihydrogen phosphate aqueous solution $(3: 17)$ flowing at $1 \mathrm{~mL} / \mathrm{min}$. Nitenpyram separated from the coexisting compounds using a column (Mightysil RP-18 GP, $4.6 \mathrm{~mm}$ i.d. $\times 150 \mathrm{mmL}, 5 \mu \mathrm{m}$, Kanto Chemical,
Tokyo, Japan) was monitored as the absorbance at $270 \mathrm{~nm}$.

\section{GC-FTD analysis for CPMA and CPMF}

For comparison, CPMA and CPMF were also determined by GC-FTD after derivatization to CPF according to the official analytical method of the Japanese Ministry of Health, Labor, and Welfare. An agricultural product sample (5g) was homogenized with $20 \mathrm{~mL}$ of acetone twice as described in Section 4, and the filtrate obtained through glass wool was treated at $50^{\circ} \mathrm{C}$ for $90 \mathrm{~min}$ and concentrated using an evaporator. The concentrated solution was kept in a $50^{\circ} \mathrm{C}$ water bath for $30 \mathrm{~min}$ after addition of $0.2 \mathrm{~mL}$ triethylamine. The solution was treated with a diatomaceous earth column and a silica gel column as for nitenpyram, described in Section 4. The difference from the pretreatment for nitenpyram was that an acetone-hexane mixture $(1: 4$, $50 \mathrm{~mL}$ ) was used instead of acetone-dichloromethane and the final concentrated eluent was $4 \mathrm{~mL}$ with acetone.

The GC instrument used was a Shimadzu GC-2010 equipped with a capillary column $(0.25 \mathrm{~mm}$ i.d. $\times 30 \mathrm{~m}, 0.25 \mu \mathrm{m}$ thickness, J\&W DB-1701, Agilent, CA). The extract sample $(2 \mu \mathrm{L})$ was injected in the splitless mode into an injector heated at $250^{\circ} \mathrm{C}$. The column oven temperature was kept at $80^{\circ} \mathrm{C}$ for $1 \mathrm{~min}$ and then increased to 180 and up to $250^{\circ} \mathrm{C}$ at the rate of $30^{\circ} \mathrm{C} / \mathrm{min}$ in both cases; temperatures of 180 and $250^{\circ} \mathrm{C}$ were maintained for $15 \mathrm{~min}$ and $20 \mathrm{~min}$, respectively. The detector temperature was set at $280^{\circ} \mathrm{C}$. 
Table 2. Recoveries of nitenpyram, CPMA, and CPMF from four kinds of agricultural samples

\begin{tabular}{|c|c|c|c|c|c|}
\hline \multirow[b]{2}{*}{ Compound } & \multirow[b]{2}{*}{ Sample } & \multirow[b]{2}{*}{ Concentration $/ \mathrm{mg} / \mathrm{kg}$} & \multicolumn{3}{|c|}{ Recovery } \\
\hline & & & $\begin{array}{c}\text { Average and RSD } / \% \\
\qquad(n=6)\end{array}$ & $\begin{array}{l}\mathrm{LOD} / \mu \mathrm{g} / \mathrm{kg} \\
(S / N=3)\end{array}$ & $\begin{array}{c}\mathrm{LOQ} / \mu \mathrm{g} / \mathrm{kg} \\
(S / N=10)\end{array}$ \\
\hline \multirow[t]{8}{*}{ Nitenpyram } & \multirow[t]{2}{*}{ Sweet pepper } & 0.005 & $98.7 \quad 4.0$ & 0.24 & 0.80 \\
\hline & & 0.5 & $103.0 \quad 1.9$ & & \\
\hline & \multirow{2}{*}{$\begin{array}{l}\text { Mandarin orange } \\
\text { (pulp without peel) }\end{array}$} & 0.005 & $93.0 \quad 3.0$ & 0.36 & 1.20 \\
\hline & & 0.5 & $103.3 \quad 3.5$ & & \\
\hline & \multirow[t]{2}{*}{ Onion } & 0.005 & $105.0 \quad 3.1$ & 0.31 & 1.05 \\
\hline & & 0.5 & $103.8 \quad 1.7$ & & \\
\hline & \multirow[t]{2}{*}{ Brown rice } & 0.005 & $99.3 \quad 2.4$ & 0.36 & 1.20 \\
\hline & & 0.5 & $103.7 \quad 3.9$ & & \\
\hline \multirow[t]{8}{*}{ CPMA } & \multirow[t]{2}{*}{ Sweet pepper } & 0.005 & $99.3 \quad 4.7$ & 0.14 & 0.47 \\
\hline & & 0.5 & $102.0 \quad 4.6$ & & \\
\hline & \multirow{2}{*}{$\begin{array}{l}\text { Mandarin orange } \\
\quad \text { (pulp without peel) }\end{array}$} & 0.005 & $99.3 \quad 3.3$ & 0.18 & 0.60 \\
\hline & & 0.5 & $101.8 \quad 3.3$ & & \\
\hline & \multirow[t]{2}{*}{ Onion } & 0.005 & $98.3 \quad 5.4$ & 0.19 & 0.64 \\
\hline & & 0.5 & $105.3 \quad 3.6$ & & \\
\hline & \multirow[t]{2}{*}{ Brown rice } & 0.005 & $93.0 \quad 5.0$ & 0.12 & 0.42 \\
\hline & & 0.5 & $95.4 \quad 7.4$ & & \\
\hline \multirow[t]{8}{*}{ CPMF } & \multirow[t]{2}{*}{ Sweet pepper } & 0.005 & $99.0 \quad 5.2$ & 0.17 & 0.56 \\
\hline & & 0.5 & $106.6 \quad 2.5$ & & \\
\hline & \multirow{2}{*}{$\begin{array}{l}\text { Mandarin orange } \\
\text { (pulp without peel) }\end{array}$} & 0.005 & $106.3 \quad 7.0$ & 0.18 & 0.58 \\
\hline & & 0.5 & $98.6 \quad 6.8$ & & \\
\hline & \multirow[t]{2}{*}{ Onion } & 0.005 & $103.7 \quad 3.1$ & 0.13 & 0.43 \\
\hline & & 0.5 & $105.0 \quad 5.5$ & & \\
\hline & \multirow[t]{2}{*}{ Brown rice } & 0.005 & $99.3 \quad 4.9$ & 0.13 & 0.44 \\
\hline & & 0.5 & $\begin{array}{ll}105.5 & 2.1\end{array}$ & & \\
\hline
\end{tabular}

\section{Results and Discussion}

\section{Chromatography analysis conditions}

In the HILIC separation, a water-rich liquid layer is formed on solid particles, and analytes move between the hydrophilic layer in the stationary phase and the mobile phase. The separation performance is largely affected by the condition of the waterrich layer, in which water is supplied from the mobile phase. Therefore, the retention time and peak height tend to vary when column equilibrium is not sufficiently achieved. Accordingly, the isocratic mode was selected, in which the composition of the mobile phase was constant and the column was equilibrated with the mobile-phase matrix.

Selection and tuning of the SRM parameters were performed by direct injection of a $1 \mathrm{mg} / \mathrm{L}$ standard solution of each analyte. The optimized MS/MS transitions as well as specific cone voltages and collision energies are summarized in Table 1. Two transitions were measured for analyte identification and confirmation, and one of them was used for quantification.

Five kinds of HILIC columns, which are explained in the Materials and Methods section, were tested for the chromatographic analysis of nitenpyram and the metabolites with distilled water-acetonitrile-500 $\mathrm{mM}$ ammonium acetate $(25: 70: 5)$ as the carrier stream. For the amide-type, sulfobetaine-type, diol-type, and triazole-type columns, the analytes did not interact well in the stationary phase, and the retention times were less than $5 \mathrm{~min}$. On the other hand, good chromatogram peaks were obtained with the non-modified silica gel column, as shown in Fig. 3. The retention times were $4.6 \mathrm{~min}$ for nitenpyram, $5.1 \mathrm{~min}$ for CPMA, and $9.4 \mathrm{~min}$ for CPMF. The estimated numbers of plates were 5100-5200 for these three compounds. Thus, a nonmodified silica gel column was chosen for the subsequent experiments.

\section{Chromatography performance}

Under optimal conditions, good performance was obtained for chromatographic analysis. Figure 3 shows the chromatograms for sweet pepper samples with and without the addition of nitenpyram, CPMA, and CPMF obtained with the non-modified silica gel column. In this method, the analytes were separated by HILIC and detected by tandem mass spectrometry, which was sensitive as well as selective. Thus, the diluted sample could be introduced to the determination system without any purification procedure. A good peak shape was obtained for each compound, and an interference peak was not observed in the real sample chromatograms.

The calibration curves for nitenpyram, CPMA, and CPMF were straight $\left(R^{2}>0.999\right)$ in the range from 0.2 to $200 \mu \mathrm{g} / \mathrm{L}$. 
Table 3. Nitenpyram, CPMA, and CPMF found in agricultural samples by HILIC-MS/MS and conventional methods

\begin{tabular}{|c|c|c|c|c|}
\hline \multirow{2}{*}{ Sample } & \multirow{2}{*}{ Compound } & \multicolumn{2}{|c|}{ Residue level ${ }^{a} / \mathrm{mg} / \mathrm{kg}$} & \multirow{2}{*}{ Ratio, \% } \\
\hline & & HILIC-MS/MS & Conventional method ${ }^{c)}$ & \\
\hline \multirow[t]{4}{*}{ Asparagus 1} & Nitenpyram & $0.162 \pm 0.0069$ & $0.155 \pm 0.0125$ & 104.5 \\
\hline & CPMA & $0.113 \pm 0.0028$ & - & - \\
\hline & CPMF & $0.191 \pm 0.0096$ & - & - \\
\hline & $\mathrm{CPF}$ & $0.267^{b)}$ & $0.274 \pm 0.0051$ & 97.4 \\
\hline \multirow[t]{4}{*}{ Asparagus 2} & Nitenpyram & $0.100 \pm 0.0044$ & $0.094 \pm 0.0028$ & 106.4 \\
\hline & CPMA & $0.045 \pm 0.0012$ & - & - \\
\hline & CPMF & $0.035 \pm 0.0010$ & - & - \\
\hline & $\mathrm{CPF}$ & $0.068^{b)}$ & $0.070 \pm 0.0065$ & 97.1 \\
\hline \multirow[t]{4}{*}{ Melon (pulp without peel) } & Nitenpyram & $0.031 \pm 0.0005$ & $0.031 \pm 0.0009$ & 100.0 \\
\hline & CPMA & $0.009 \pm 0.0005$ & - & - \\
\hline & CPMF & $0.033 \pm 0.0018$ & - & - \\
\hline & $\mathrm{CPF}$ & $0.038^{b)}$ & $0.036 \pm 0.0021$ & 105.6 \\
\hline
\end{tabular}

a) Results of residue level are indicated as average and standard deviation of values obtained three times. ${ }^{b}$ CPMA and CPMF values are measured in terms of CPF value. ${ }^{c}$ Nitenpyram was measured by HPLC-UV and CPF was by GC-FTD. ${ }^{d)}$ Ratios are HILIC-MS/MS data to conventional methods data.

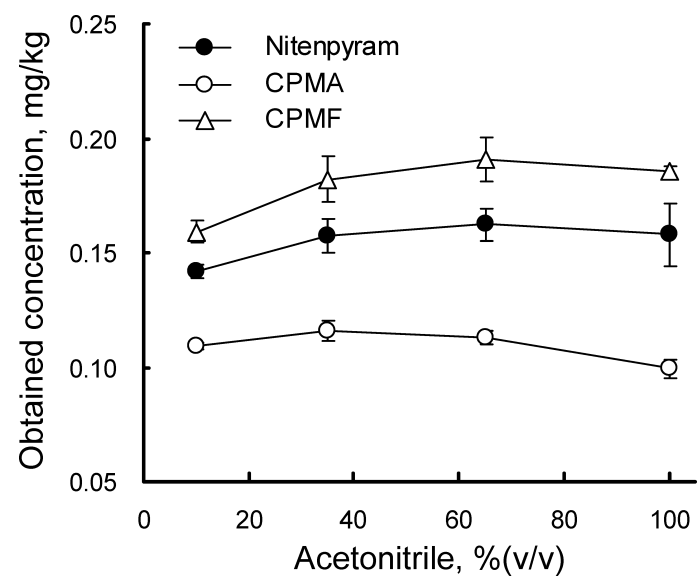

Fig. 4. Effect of acetonitrile concentration in extraction solvent on nitenpyram, CPMA, and CPMF extracted from an asparagus sample.

The linear range corresponded to $0.002-2 \mathrm{mg} / \mathrm{kg}$ for $5 \mathrm{~g}$ of agricultural product sample. From the signal-to-noise ratio $(S / N)$, the limits of detection (LOD, $S / N=3$ ) and limits of determination (LOQ, $S / N=10$ ) were obtained as shown in Table 2: LODs and LOQs were $0.24-0.36$ and $0.80-1.20 \mu \mathrm{g} / \mathrm{kg}$ for nitenpyram, $0.12-0.19$ and $0.42-0.64 \mu \mathrm{g} / \mathrm{kg}$ for CPMA, and $0.13-0.18$ and $0.43-0.58 \mu \mathrm{g} / \mathrm{kg}$ for CPMF, respectively. Accordingly, the presented method had enough sensitivity to determine $1 / 10$ of the uniform limit $(10 \mu \mathrm{g} / \mathrm{kg})$ determined by the Japanese Ministry of Health, Labor, and Welfare.

\section{Examination of extraction solvent}

Contaminated asparagus was treated with different water/acetonitrile mixtures for the extraction of nitenpyram, CPMA, and CPMF. The obtained compound concentrations were plotted against the acetonitrile content in Fig. 4. The nitenpyram and CPMF values increased with an increase in the acetonitrile concentration up to $65 \%$. This suggested that a higher organic solvent ratio was required for effective extraction from agricultural samples. At 100\%, however, the extracted CPMA amount decreased, probably due to the high polarity of CPMA or conversion to CPMF, as mentioned in the Introduction. ${ }^{2)}$ Accordingly, the ratio of acetonitrile and water was adjusted at 65:35 for the subsequent experiments, such as the recovery test and comparison with the conventional methods for contaminated samples.

\section{Analysis of agricultural products}

LC-MS/MS analysis sometimes gives errors due to ion suppression or ion enhancement in the ionization if the sample contains a matrix in high content. ${ }^{2)}$ Ion enhancement was observed in the HILIC-MS/MS analysis of the agricultural samples. For example, the SRM chromatogram peak areas of matrix-matched standard solutions increased by $103-126 \%$ for nitenpyram, 110-129\% for CPMA, and 103-125\% for CPMF relative to standard solutions. Therefore, recovery tests were performed for samples containing a variety of matrices, namely, samples containing a high content of chlorophyll (sweet pepper), acidic samples (mandarin orange), samples highly containing sulfide groups (onion), and samples rich in lipid (brown rice). For all samples, the added concentrations were 0.005 and $0.5 \mathrm{mg} / \mathrm{kg}$, and good chromatographic peaks were obtained for all samples without an interference peak. The recovery tests were repeated 6 times for each sample. The results of recovery tests are shown in Table 2. Recoveries at a lower concentration $(0.005 \mathrm{mg} / \mathrm{kg})$ were $93.0-105.0 \%$ for nitenpyram, $93.0-99.3 \%$ for CPMA, and 99.0-106.3\% for CPMF. When the chemicals were added at a higher concentration $(0.5 \mathrm{mg} / \mathrm{kg})$, the results were $103.0-103.8 \%$ for nitenpyram, 95.4-105.3\% for CPMA, and 98.6-106.6\% for CPMF. Thus, the extraction was quantitative for a variety of samples with different concentrations. For dry samples, such as brown rice, it is reportedly requred to inject water into samples before organic solvent extraction to cause the tissues to 
swell. ${ }^{12,13)}$ On the other hand, in this method, although a water injection was not used, good recovery was obtained by using a water-mixed solvent even in the extraction from brown rice. In our experiments without a water injection, the extraction efficiency of real residue from dry samples is quantitatively uncertain. The inter-day deviations for nitenpyram, CPMA, and CPMF were 7.0, 3.9, and 3.9\%, respectively, which were obtained for sweet pepper tested $(0.5 \mathrm{mg} / \mathrm{kg}, n=1)$ for 6 days.

In order to confirm the applicability of this method, we searched for samples containing the compounds that we sought to evaluate. From 60 samples obtained in the Kumamoto prefectural research center and retail stores, two asparagus samples and a melon sample were found to contain nitenpyram, CPMA, and CPMF. For these three samples, the compounds were analyzed by the presented water/acetonitrile extraction followed by HILIC-MS/MS and the conventional methods. In the conventional methods, nitenpyram was determined by HPLC-UV, and CPMA and CPMF were converted to CPF and analyzed by GCFTD as the sum of the two compounds. Those results are compared in Table 3. The ratio of the HILIC-MS/MS data against the conventional method was $100.0-106.4 \%$ for nitenpyram and 97.1-105.6\% for the sum of CPMA and CPMF (CPF). The data by HILIC-MS/MS agreed well with those by the conventional methods from highly contaminated asparagus to the less contaminated melon sample. Furthermore, the three compounds were determined simply and simultaneously by the proposed method, whereas two complicated analytical methods were required in the conventional methods. In addition, the concentrations of CPMA and CPMF were given individually only in the proposed method. This showed that HILIC-MS/MS is a useful and reliable analytical method for nitenpyram and its metabolites contained in real agricultural product samples.

\section{Conclusions}

A simple analytical method was developed for nitenpyram, CPMA, and CPMF individually using HILIC-MS/MS, which is advantageous for highly polar compounds. Recovery tests and real sample analyses were performed, and good results were obtained for all samples tested. Purification was not needed because HILIC-MS/MS was a highly selective determination method and pretreatment of the sample is simple and less timeconsuming, requiring no specialized skills. In addition, those three compounds could be extracted simultaneously with a throughput of 6 samples/hr, which was one seventh of conventional analytical procedure according to the official analytical method of the Japanese Ministry of Health, Labor, and Welfare. The present method analyzes CPMA and CPMF separately, and the metabolism of nitenpyram to these compounds can be monitored. The method is expected to be of use in the research of the metabolism dynamics of the pesticide. Moreover, it is environmentally safer because it requires only a small amount of organic solvent. The proposed method is useful for the analysis of nitenpyram, CPMA, and CPMF contained in agricultural products.

\section{References}

1) Notification of the Japanese Ministry of Health, Labor, and Welfare, Syoku-An No. 0124001, Jan. 24, 2005.

2) H. Kobayashi: Bunseki Kagaku 58, 985-997 (2009) (in Japanese).

3) Y. Tsumura, Y. Nakamura, Y. Tonogai, Y. Kakimoto, Y. Tanaka and T. Shibata: Shokuhin Eiseigaku Zasshi 39, 127-134 (1998) (in Japanese).

4) T. Yoshida and T. Tobino: Annual Report of Kumamoto Prefectural Institute of Public Health and Environmental Science 39, 32-35 (2009) (in Japanese).

5) K. Fukushima, M. Masunaga, Y. Miyahara and T. Tobino: Annual Report of Kumamoto Prefectural Institute of Public Health and Environmental Science 37, 36-39 (2007) (in Japanese).

6) A. J. Alpert: J. Chromatogr. A 499, 177-196 (1990).

7) Y. Iwasaki, K. Inoue, R. Ito, Y. Yoshimura, K. Saito and H. Nakazawa: Bunseki Kagaku 54, 135-142 (2005) (in Japanese).

8) T. Ikegami, H. Takubo and N. Tanaka: Chromatogr. 29, 1-6 (2008) (in Japanese).

9) C. Lamouroux, G. Foglia and G. Le Rouzo: J. Chromatogr. A 1218, 3022-3028 (2011).

10) P. Jandera: Anal. Chim. Acta 692, 1-25 (2011).

11) T. Yoshida, H. Hamada, H. Murakawa, H. Yoshimoto, T. Tobino and K. Toda: Anal. Sci. 28, 179-182 (2012).

12) S. Nemoto: Shokuhin Eiseigaku Zasshi 51, 349-359 (2010) (in Japanese).

13) M. Saka, K. Iijima, Y. Koma, M. Fujita, Y. Odanaka and Y. Kato: J. Pestic. Sci. 31, 431-434 (2006) (in Japanese). 